\title{
Improving the selection of MPRs in OLSR protocol: a survey of methods and techniques
}

\author{
Omar Barki ${ }^{1}$, Zouhair Guennoun ${ }^{2}$, Adnane Addaim $^{3}$ \\ ${ }^{1,2}$ Smart Communications, E3S Research Center, EMI, Mohammed V University, Morocco \\ ${ }^{3}$ ISET Laboratory, ENSA, Ibn Tofail University, Morocco
}

\begin{tabular}{|c|c|}
\hline Article Info & ABSTRACT \\
\hline Article history: & Multi Point Relays (MPRs) are those nodes that are calculated and \\
\hline Received Jan 30, 2019 & $\begin{array}{l}\text { determined by the Optimized Link State Routing protocol (OLSR) in order to } \\
\text { minimize and avoid overload inside the Mobile Ad hoc Network (MANET). }\end{array}$ \\
\hline Revised Jul 26, 2019 & In this paper, we will present a synthetic study of many techniques and \\
\hline Accepted Aug 29, 2019 & $\begin{array}{l}\text { methods for calculating and selecting the MPR nodes using a set of criteria } \\
\text { namely energy, mobility, bandwidth, the quality of links, etc. The result of }\end{array}$ \\
\hline Keywords: & $\begin{array}{l}\text { this study shows that most techniques consider a limited number of metrics } \\
\text { for selecting the MPR nodes and therefore they are insufficient to allow the }\end{array}$ \\
\hline $\begin{array}{l}\text { MANET } \\
\text { MPR }\end{array}$ & $\begin{array}{l}\text { OLSR protocol to be quite complete and efficient because several metrics } \\
\text { can occur at the same time in the real execution environment. }\end{array}$ \\
\hline
\end{tabular}

OLSR

Wireless network

\section{Corresponding Author:}

Omar Barki,

Smart Communications (ERSC), Ecole Mohammadia d'ingenieur,

Mohammed V University, Rabat.

Avenue Ibn Sina BP765 Agdal, Rabat, Morocco.

Email: obarkiomar@gmail.com

\section{INTRODUCTION}

The OLSR (Optimized Link State Routing) is a proactive routing protocol dedicated to MANET networks (Mobile Ad hoc Network) for establishing paths between network nodes. The routing process of this protocol is mainly based on the MPR (Multi Point Relay) selection technique in order to reduce the traffic load of the network and also to minimize the path to the destination [1, 2]. the MPRs search procedure launched by the NPC (Node Performing the Computation) makes it possible to detect a set of nodes able to transmit its messages and its data to other nodes far from its range and therefore cover the entire network. The computation of the MPR by the standard algorithm RFC3626 [3] presented below remains basic and insufficient because it does not take into account all the characteristics of the node and the network, for a thorough study of this problem we compared several approaches proposed by researchers in order to improve the quality of service $[4,5]$ and security level $[6,7]$ of this protocol against attacks $[8,9]$.

In the next section, we will present a brief description of a set of OLSR improved versions and approaches especially the MPRs selection technique, then in the third section, we will draw up a summary table of the comparative study and finally, we will conclude our study by analyzing the obtained results.

\section{IMPROVED VERSIONS OF THE OLSR PROTOCOL (MPR)}

In this section, we will present a brief description of some techniques used for improving MPR selection algorithm in order to reduce the number of transmitted messages, optimize data transfer time between source and destination nodes and also to reduce the energy consumption for increasing the lifetime of the network. 


\subsection{OLSR}

According to RFC3626 [3], the technique used to calculate these MPRs is based on the symmetrical connections between the NPC node and its first and second neighbor nodes, is given by the following algorithm:

$N$ : The subset of the node's neighbors, which are neighbors of the interface $I$.

N2 : The set of 2-hop neighbors reachable from the interface $\boldsymbol{I}$, excluding:

a. the nodes only reachable by members of $N$ with willingness WILL_NEVER

b. the node performing the computation

c. all the symmetric neighbors: the nodes for which a symmetric link to this node exists on some interface.

$D(y)$ : The degree of a 1-hop neighbor node $\boldsymbol{y}$ (where $\boldsymbol{y}$ is a member of $N$ ), is defined as the number of symmetric neighbors of node $\boldsymbol{y}$, EXCLUDING all members of $\boldsymbol{N}$ and EXCLUDING the node performing the computation.

The proposed heuristic is as follows:

1. Start with an MPR set made of all members of $N$ with $N$ _willingness equal to WILL_ALWAYS

2. Calculate $\mathrm{D}(\mathrm{y})$, where y is a member of $\boldsymbol{N}$ for all nodes in $\boldsymbol{N}$.

3. Add to the MPR set those nodes in $\mathrm{N}$, which are the only nodes to provide reachability to a node in $N 2$. For example, if node $\mathrm{b}$ in $N 2$ can be reached only through a symmetric link to node a in $N$, then add node a to the MPR set. Remove the nodes from $N 2$ which are now covered by a node in the MPR set.

4. If there are nodes in $N 2$ which are not covered by at least one node in the MPR set :

a. For each node in $N$, we calculate the reachability, i.e., the number of nodes in $N 2$ which are not yet covered by at least one node in the MPR set, and which are reachable through this 1-hop neighbor;

b. We select as a MPR the node with highest $N$ willingness among the nodes in $N$ with non-zero reachability. In case of multiple choices, we select the node which provides reachability to the maximum number of nodes in $N 2$. In case of multiple nodes providing the same amount of reachability, we select the MPR node as the one where $\mathrm{D}(\mathrm{y})$ is greater. Remove the nodes from $N 2$ which are now covered by a node in the MPR set.

\subsection{L-OLSR}

In this version [10], the MPR selection algorithm takes into account the angle between two lines : the line composed of NPC node and node with the highest accessibility level noted: NA and the line composed of NPC node and candidate node for MPR noted: NC. If the angle is close to 90, 180 or 270, this node is considered MPR for the NPC node. The calculation of the NC-NPC-NA angle is made from the distances NPC-NA, NPC-NC and NA-NC (determined from the coordinates of each node) by the following formula:

$$
\mathrm{x}=\operatorname{arcos}([(\mathrm{NPC}-\mathrm{NA}) 2+(\mathrm{NPC}-\mathrm{NC}) 2-(\mathrm{NA}-\mathrm{NC}) 2] / 2 * \mathrm{NA}-\mathrm{NC})
$$

The result of the simulation on NS3 shows that the number of packets sent by standard OLSR is large compared to the number sent by L-OLSR but the number of packets received is almost the same for both OLSR versions, hence the number of lost packets for L-OLSR is minimal compared to OLSR. finally, the proposed L-OLSR approach (based on locating nodes for the selection of MPRs) is more efficient in terms of PDR, and therefore it improves the ability to transmit messages and data.

\subsection{BW-OLSR}

This is a modified version of the RFC3626 MPR Selection Algorithm (SA MPR) in which the authors added the bandwidth as a parameter to consider for path computation between the source and destination node [11]. The path to take among the possible paths computed by OLSR via the MPRs of the NPC node is the one which contains the maximum of the nodes which have a wider bandwidth without this path necessarily being the shortest. The simulation carried out on OPNET for both versions OLSR and BW-OLSR showed that the latter presented a number of MPRs greater than that calculated by OLSR and consequently a significant increase in rate in the network.

\subsection{DF-OLSR}

According to [12], the authors have tried to improve MPRs selection in terms of energy consumption and at the same time to secure it against nodes that are suspicious by isolating them from the rest of the network. The proposed algorithm is based on the exchange of three new messages namely: VOTEFOR and VOTERPL which mark every time the sequence of nodes N1 (1st neighborhood) by nodes N2 (2nd neighborhood) accessible by the nodes of N1. The nodes of N1 having the highest number of votes 
(VOTERPL maximum) is considered as MPR. The third message "INFO" is sent to N2 nodes to inform them of their MPR. This approach allowed reducing the energy consumption and quickly detecting the nodes using DOS to attack the network.

\subsection{IOLSR}

The idea is to detect malicious nodes by using the number of HELLO messages sent, then block them for some time [13]. The solution consists in determining a threshold of number of Hello messages sent per unit of time knowing that the periodicity of this message is fixed at 2 seconds. Indeed, if the calculated number is higher than the normal value then the node is considered suspicious and it is blocked for a certain time. This approach helps reduce congestion in the network and prevent malicious nodes.

\subsection{PB-OLSR}

The objective of the PB-OLSR (Performance Based OLSR) is to improve exchanges and services in the MANET network via MPRs that are more efficient and less energy-consuming by relying on a measurement model of the performance and confidence level of each node [14]. Indeed, firstly for the computation of the node performance, the author used the Multi Creteria Decision Analysis (MCDA) method and ROC (Rank Order Centroid) precisely on a set of criteria of this node (residual energy, RAM, CPU , ....) in order to determine the weight of each of these metrics. Secondly, to evaluate the trust of the node, he used an algorithm that relies on HELLO, TC messages and data messages routed through this node using the ROC technique to calculate the weight of each message. When receiving control messages or data messages, the algorithm increments the confidence value associated to the node. If a malicious behavior is detected, the algorithm will decrement the confidence value. The result of the simulation on NS3 has been shown that this approach allows:

a. Reducing the impact of malicious nodes by calculating trust.

b. Reducing the broadcast traffic in the network

c. Maximizing the routing lifetime by avoiding nodes with poor performance being elected as MPRs.

\subsection{EM-OLSR}

The selection of MPRs by the OLSR protocol is reinforced in this approach by calculating the value of the Willingness parameter from two metrics, namely the energy and mobility of the node [15]. Each node calculates its residual energy and its moving speed (supposing that each node is equipped with GPS to determine its coordinates) and it deduces from it the value of Willingness which can be willigness_default, willigness_low or willigness_high according to the result of the following algorithm:

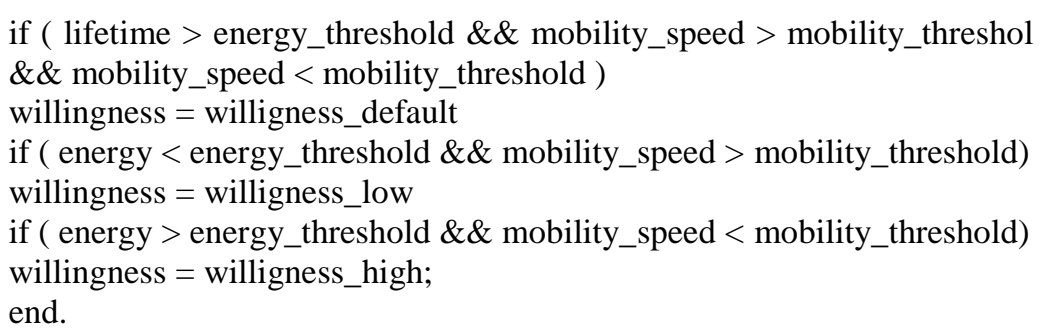

The result of the simulation performed on NS2 simulator shows that the EM-OLSR version gives better results compared to the OLSR standard protocol in terms of throughput, lost packets and energy consumption.

\subsection{W-OLSR}

W-OLSR [16] is an OLSR standard extension in which the author has added for the selection of MPRs another parameter called Weihgted-MPR calculated from Residual Energy, Signal Strength and Transmission delay by the following formula:

$$
\text { Weighted MPR }=X * \text { Residual Energy }+Y * \text { Signal Strength }-Z * \text { Transmission delay }
$$

Where $\mathrm{X}, \mathrm{Y}$ and $\mathrm{Z}$ are constants, if Weighted MPR < = weight_threshold then the node is considered MPR. At the source node the HELLO message is sent with the value of the residual energy and transmission time, and in reception the quality of this link (bad or good) is determined by measuring the residual energy, delay of transmission (sending time and current times) and the signal strength. The comparison of OLSR standard 
with W-OLSR showed that the latter is more efficient compared to OLSR in terms of throughput and number of lost packets (PDR).

\subsection{EOLSR, EOLSR-EC, EOLSR-RE}

According to [17], to guarantee a long MANET network lifetime, the OLSR version is reinforced by the addition of energy metric that produce the EOLSR (OLSR for energy consumption) version, which has made it possible to minimize the energy consumption and optimize the data transfer path calculation by modifying the HELLO and TC message formats by adding two types of parameters namely residual energy (RE) and consumed energy(EC), thus two versions for EOLSR have been established namely EOLSR-RE and EOLSR-EC. This approach consists in setting the thresholds of RE and EC and checking the following test: If the residual energy of the node is lower than the threshold value, the node is set to LOW-MPR-WILL, otherwise it is HIGH-MPR-WILL. And if the energy consumed by the node is less than the threshold value, the node takes the value HIGH-MPR-WILL otherwise it takes the value LOW-MPR-WILL. From the simulation result of this approach, the author noticed that the OLSR-RE is the best protocol in terms of energy efficiency compared to EOLSR-EC and OLSR.

\subsection{M-OLSR}

OLSR is modified so that each node in the network can choose its strategy of "Update" or "Not update" the HELLO and TC messages in order to minimize the cost of path to borrow in terms of number of jump and energy [18]. This technique depends on the energy capacity of the intermediate nodes, if it reaches the threshold (given by the user), then it chooses another path. The evaluation of the performance of this approach is performed using various parameters namely "end to end delay", "overhead routing" and "residual energy". The results obtained from this simulation show that the proposed MOLSR algorithm offers a significant reduction of load of messages in the network and the energy consumption and also it presents a small reduction in the average throughput.

\subsection{OLSR-ETX-ML-MD}

The author tried to compare the three versions of OLSR, namely ETX, ML and MD [19], knowing that: OLSR-ETX is based on the link quality in terms of sending and receiving HELLO messages, by the probability calculation (number of messages sent by $X$ to $Y$ / number of messages received by $Y$ from $X$ ) for each node and the formula ETX is given by: ETX $=1 / \mathrm{P}(\mathrm{X}) * \mathrm{P}(\mathrm{Y})$. So, for choosing the shortest path, we consider the path which has the sum of the smallest ETX.

OLSR-ML is the basis for calculating ETX. This is the product called PLINK $=\mathrm{P}(\mathrm{X}) * \mathrm{P}(\mathrm{Y})$, finds the link where the number of lost packets is smaller. OLSR-MD is based on the measurement of transmission delay between the nodes calculated from the routing table. The shortest path is the path for which the delay is smaller. The comparison of this version has shown that OLSR-ETX gives more satisfaction in terms of evaluation parameters (end-to-end delay, Throughput ...).

\subsection{MOB-2--OLSR}

The proposed Mob-2-OLSR protocol is an improvement on the two previous versions: OLSR and Mob-OLSR in order to limit the effects produced by the mobility of neighboring nodes and reduce the rate of packet loss and the value "End-to-end delay" [20]. For this purpose, the authors introduce metrics that calculate the mobility of neighboring nodes in the process used for the selection of MPR by giving priority to less mobile candidates. The proposed protocol Mob-2-OLSR provided, in most cases, the best results in terms of PDR, delay and throughput compared to other versions.

\subsection{EDCR-OLSR}

According to [21], the authors took advantage of the idea used in the latest generation of microprocessors. Instead of using a single processor with high execution speed and thus highpower consumption, it would be preferable to use the processor with several cores in the same chip with an overall speed equivalent to the first processor and whose Energy consumption is reduced. Thus, by applying this idea to the OLSR protocol, the retransmission traffic control task is distributed over several MPRs. As results of this technique, the authors remarked a gain in terms of energy consumption and packet loss ratio.

\subsection{OLSR-AAD}

The technique proposed in [22] consists in allowing each node the possibility to keep the maximum possible MPR. The authors have changed the order of selection of MPRs so that they introduce a new metric "Average Age of Death" based on the lifetime of the node already selected as MPR which depends on several 
criteria ie (Node mobility, network density, etc.). This approach was satisfactory in terms of PDR, delay and throughput.

\subsection{EEPR-OLSR}

In the article [23], the author tries to improve the MANET network lifetime by maintaining routing path availability and minimizing power consumption in the OLSR protocol. The upgrade version is called EEPR_OLSR, it has presented significant results compared to standard OLSR. The author used GPS to retrieve information on the nodes in order to calculate the most stable path by Link Expiration Time (LET) giving by this formula:

$$
\operatorname{LET}(i, j)=\frac{-(a b+c d)+\sqrt{\left(a^{2}+c^{2}\right) r^{2}-(a d-b c)^{2}}}{a^{2}+c^{2}}
$$

where

$$
\begin{aligned}
& a=v_{i} \cos \theta_{i}-v_{j} \cos \theta_{j}, \quad b=x_{i}-x_{j}, \\
& c=v_{i} \sin \theta_{i}-v_{j} \sin \theta_{j}, d=y_{i}-y_{j} .
\end{aligned}
$$

For this EEPR_OLSR version, the simulation on NS3 has given a high PDR and life time results compared to OLSR Standard.

\subsection{EEM-OLSR}

The main idea given by the authors of the article [24] is to use the technique of energy efficiency by choosing the shortest available path. Otherwise, we take the other shortest available alternative path in the case where the first path goes down. For this reason, the authors have modified the structure of the routing table by adding information that records the different paths from the same source to the same destination from the topology table. The comparison of this new version of OLSR with the OLSR standard, according to the parameters namely Throughput, Packet Delivery Ratio, Energy Efficiency, Normalized Routing load and End-to-End Delay, has shown that this approach has presented a significant optimization in terms of time and energy.

\subsection{DCFM-OLSR}

The author has attempted to use the Denial Contradictions with Fictitious Node Mechanism (DCFM) technique to address possible attacks on OLSR protocol performance [25]. This technique is based on the same strategy followed by the attack itself in order to avoid it. It creates virtual nodes and uses data from the victim node to protect the network. The result of the proposed approach shows that it avoids a large number of attacks and therefore a more reliable data exchange could be set up between nodes.

\subsection{OPE-OLSR}

In the paper [26], the authors proposed a new routing approach in the OLSR protocol based on the energy consumption for selecting the MPR nodes in order to increase the lifetime of the MANET network. The main idea is to try to privilege the nodes in N1 having higher energy to be an MPR because the MPR node consumes more energy than other non-MPR nodes. If several nodes have the same value of energy, we choose the MPR node which covers more nodes in N2. The proposed algorithm is as follow:

a. Let assume X is a node which wants to compute MPR set. N1 is a set of X's 1-hop neighborhood and N2 is a set of X's 2-hop neighborhood.

b. If (Set N1 is empty)

\{Wait until topology changes Goto step 1\}

Else

\{

If (Are there two or more nodes offering the same maximum energy)

\{ Select node $\mathrm{i}$ in $\mathrm{N} 1$ which covers maximum nodes

(highest degree) in $\mathrm{N} 2$

\}

Else $\{$ Select node i which has highest Energy

\}

c. Node in N2 has symmetric link with node i of N1

Int J Elec \& Comp Eng, Vol. 10, No. 1, February 2020 : 288 - 295 
d. Choose node $\mathrm{i}$ in $\mathrm{N} 1$ as MPR

e. Delete the nodes of N2 which are symmetrically connected with node $\mathrm{i}$ in $\mathrm{N} 1$

f. If (Set N2 is empty) $\{$ S: MPR Set of X\}

Else

\{Consider node i in N1 which has Max Energy and not yet considered as MPR

Remaining nodes in $\mathrm{N} 2$ has symmetric link with node $\mathrm{i}$ of $\mathrm{N} 1$

Goto step 4.\}

\section{STUDY AND RESULTS}

The different optimization approaches of calculation of the MPR are cited in Table 1. The study of those approaches allowed us to note the following remarks:

a. Most approaches have focused on energy in comparison to other parameters (Mobility, security, bandwidth).

b. Most approaches deal with one, two or three parameters at a time without taking into account the impact of the others.

c. The consideration of the characteristics of the material is almost null in the studies carried out by the authors.

d. We also note the absence of mathematical models in several studies approaches

e. The changes made by the authors are often done on the algorithm of selection of the MPRs and the formats of messages HELLO and TC.

f. The study of the dependence between the parameters is not taken into account, knowing that the change of one parameter can affect the others.

g. The most common type of mobility used in the simulation is Random Way Point compared to Random Direction, Manhattan Grid, Freeway Point and others.

h. The simulations were performed with NS2 tool compared to NS3.

i. Lack of the reaction study of this protocol by acting on several parameters at a same time.

Table 1. Comparison between MPR selection approaches based on the authors focuses axes

\begin{tabular}{|c|c|c|c|c|c|c|c|c|c|c|c|c|c|c|}
\hline & 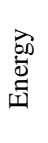 & 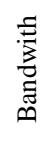 & 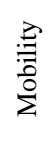 & 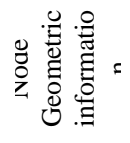 & 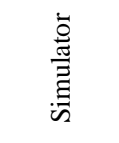 & 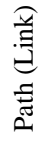 & 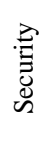 & $\frac{\circ}{\frac{0}{9}}$ & $\stackrel{0}{H}$ & 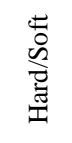 & 竭 & 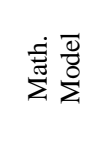 & 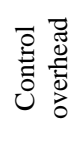 & 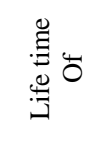 \\
\hline OLSR & & & & & & $\mathrm{X}$ & & $\mathrm{X}$ & $\mathrm{X}$ & $\mathrm{S}$ & RWP & & & \\
\hline L-OLSR & & & & $\mathrm{X}$ & NS3 & $\mathrm{X}$ & & & & S & $\begin{array}{c}\text { RD2dM } \\
\text { M }\end{array}$ & & & \\
\hline BW-OLSR & & $\mathrm{X}$ & & & OPNET & $X$ & & & & S & & $\mathrm{X}$ & & \\
\hline DF-OLSR & $\mathrm{X}$ & & & & & & $\begin{array}{l}X \\
X\end{array}$ & & & $\mathrm{~S}$ & & & & \\
\hline $\begin{array}{l}\text { IOLSR } \\
\text { PB-OLSR }\end{array}$ & & & & & NS2 & $\begin{array}{l}X \\
X\end{array}$ & $\begin{array}{l}X \\
X\end{array}$ & $\begin{array}{l}X \\
X\end{array}$ & $\mathrm{X}$ & $\begin{array}{c}\mathrm{S} \\
\mathrm{H}, \mathrm{S}\end{array}$ & $\begin{array}{l}\text { RWP } \\
\text { RWP }\end{array}$ & MCDA & & \\
\hline EM-OLSR & $\mathrm{X}$ & & $\mathrm{X}$ & GPS & NS2 & $X$ & & $\mathrm{X}$ & & & $\begin{array}{l}\text { RWP, } \\
\text { MG, FP }\end{array}$ & & & \\
\hline EOLSR-EC & $\mathrm{X}$ & & & & & $X$ & & $\mathrm{X}$ & $X$ & S & RWP & & & \\
\hline W-OLSR & $\mathrm{X}$ & & $X$ & & & $X$ & & $\mathrm{X}$ & & $\mathrm{S}$ & RWP & & $\mathrm{X}$ & \\
\hline MOLSR & $X$ & & & & NS3 & $X$ & & $X$ & $\mathrm{X}$ & $\mathrm{S}$ & & & $\mathrm{X}$ & \\
\hline OLSR-ML & & $X$ & & & NS2 & $X$ & & $\mathrm{X}$ & & $\mathrm{S}$ & & & $\mathrm{X}$ & \\
\hline OLSR-MD & & $X$ & & & NS2 & $X$ & & $X$ & & $\mathrm{~S}$ & & & $\mathrm{X}$ & \\
\hline OLSR-ETX & & $\mathrm{X}$ & & & NS2 & $X$ & & $\mathrm{X}$ & & $S$ & & & $\mathrm{X}$ & \\
\hline $\begin{array}{c}\text { MOB-2- } \\
\text { OLSR }\end{array}$ & & & $\mathrm{X}$ & & NS2 & $\mathrm{X}$ & & & & S & RWP & & & \\
\hline $\begin{array}{l}\text { EDCR- } \\
\text { OLSR }\end{array}$ & $\mathrm{X}$ & & & & NS2 & $X$ & & & & $\mathrm{~S}$ & RWP & & & \\
\hline $\begin{array}{l}\text { OLSR- } \\
\text { AAD }\end{array}$ & & & & & NS2 & $X$ & & & & $\mathrm{~S}$ & RWP & & & MPR \\
\hline $\begin{array}{l}\text { EEPR- } \\
\text { OLSR }\end{array}$ & $\mathrm{X}$ & & & GPS & NS3 & $\mathrm{X}$ & & & & $\mathrm{S}$ & $\begin{array}{c}\text { R.Walk- } \\
\text { based }\end{array}$ & $\mathrm{X}$ & & Network \\
\hline EEM-OLSR & $\mathrm{X}$ & & & & NS2 & $X$ & & $X$ & $X$ & S & R.D & & $\mathrm{X}$ & \\
\hline $\begin{array}{c}\text { DCFM- } \\
\text { OLSR }\end{array}$ & & & & & & $\mathrm{X}$ & $\mathrm{X}$ & $\mathrm{X}$ & & S & & & $\mathrm{X}$ & \\
\hline OPE-OLSR & $\mathrm{X}$ & & & & NS2 & $X$ & & & & $\mathrm{~S}$ & & & & Network \\
\hline
\end{tabular}




\section{CONCLUSION}

In this article, we have attempted to present a survey study of a set of approaches and techniques concerning the changes made to the OLSR protocol for improving the MPR selection technique and for maintaining the life time of the network as long as possible by allowing the network nodes more reliable exchanged data. This survey made it possible to know the impact of metrics to select the MPR nodes and calculate an optimal path from source node to destination node. Also, this survey allows knowing the orientations of the researchers and their concerns. Finally, in this article, we have given a set of remarks on the OLSR protocol execution environment that can open new research subject and new innovative ideas in this field.

\section{REFERENCES}

[1] O. Barki, et al., "Formal verification of MPR selection algorithm in the OLSR protocol," 10th International Conference, Intelligent Systems: Theories and Applications (SITA), pp. 1-5, 2015.

[2] O. Barki, et al., "Comparative study of MPR selection algorithms based on Statistical Model Checking," $5^{\text {th }}$ International Conference on Multimedia Computing and Systems (ICMCS'16), IEEE Conference, 2016.

[3] T. Clausen and P. Jacquet, "Optimized Link State Routing Protocol (OLSR)," Project Hipercom, INRIA, RFC3626, 2003.

[4] Alamsyah, et al., "MPR selection to the OLSR quality of service in MANET using minmax algorithm," International Journal of Electrical and Computer Engineering (IJECE), vol. 9, no. 1, pp. 417-425, 2019.

[5] S. Sahu and S. Sharma, "Secure and proficient cross layer (SPCL) QoS framework for mobile ad-hoc network," International Journal of Electrical and Computer Engineering (IJECE), vol. 9, no. 4, pp. 2603-2613, 2019.

[6] A. Ahmad and S. Ismail, "User Selective Encryption Method for Securing MANETs," International Journal of Electrical and Computer Engineering (IJECE), vol. 8, no. 5, pp. 3103-3111, 2018.

[7] K. R. Rao, et al., "An Effective Data Privacy Mechanism through Secure Session Key Exchange Model for MANET," International Journal of Electrical and Computer Engineering (IJECE), vol. 8, no. 5, pp. 3267-3277, 2018.

[8] Y. Khamayseh, et al., "Intelligent black hole detection in mobile AdHoc networks," International Journal of Electrical and Computer Engineering (IJECE), vol. 9, no. 3, pp. 1968-1977, 2019.

[9] N. Panda and B. K. Pattanayak, "Analysis of Blackhole Attack in AODV and DSR," International Journal of Electrical and Computer Engineering (IJECE), vol. 8, no. 5, pp. 3092-3102, 2018.

[10] W. Anbao and Z. Bin, "Improving MPR Selection Algorithm in OLSR Protocol Based on Node Localization Technology," Journal of networks, vol. 9, 2014.

[11] D. Moad, et al., "Improving the Quality of Service Routing in OLSR Protocol," International Conference Communications and Information Technology (ICCIT), 2012.

[12] G. Ezhilarasi, et al., "Improving MPR selection algorithm in olsr Protocol based on dos free transmission in MANET MPR selection process," International Journal of Technology and Engineering System (IJTES), vol 7, pp. 239-243, 2015.

[13] J. Bavaliya, et al., "An Improved OLSR Protocol IOLSR to Detect and Prevent Flood Attack over MANET," IJESC, vol. 6, 2016.

[14] M. Dyabi, et al., "PB-OLSR: Performance Based OLSR," IJCSNS International Journal of Computer Science and Network Security, vol. 15, 2015.

[15] L. Fatima and E. Najib, "Energy and mobility in OLSR routing protocol," Cyber Journals: Multidisciplinary Journals in Science and Technology, (JSAT), 2012.

[16] L. S. Anandrao and J. Y. Amey, "Comparison of OLSR and Energy Conserved OLSR," International Journal of Technical Research and Applications, vol. 2, pp. 05-08, 2014.

[17] Mohit and S. Pal, "Stable MPR Selection in OLSR for Mobile Ad-Hoc Networks," (IJCSIT) International Journal of Computer Science and Information Technologies, vol. 6, pp. 5121-5125, 2015.

[18] A. K. Jaiswal and S. Tiwari, "Modified OLSR (MOLSR) Protocol for improving optimal route selection with Dynamic MPR selection in Mobile Adhoc Network," IJSRSET, vol. 1, 2015.

[19] A. Pandey and M. Baliyan, "Performance Analysis of OLSR and Modified Version of OLSR-ETX/MD/ML in Mesh Networks," International Journal of Computer Science \& Communication Networks, vol. 2, pp. 268-271.

[20] A. Ouacha, et al., "OLSR protocol enhancement through mobility integration," 10th IEEE International Conference in Networking, Sensing and Control (ICNSC), pp. 17-22, 2013.

[21] A. Ouacha, et al., "Proactive routing based distributed energy consumption," Intelligent Systems: Theories and Applications (SITA), 2013 8th International Conference on, pp. 1-5, 2013.

[22] A. Ouacha, et al., "New Mobility Metric based on MultiPoint Relay Life Duration," presented at the Proceedings of the International Conference on Signal Processing and Multimedia Applications and Wireless Information Networks and Systems, Rome, Italy, 2012.

[23] A. Sahnoun, et al., "EEPR-OLSR: An Energy Efficient and Path Reliability Protocol for Proactive Mobile Ad-hoc Network Routing," International Journal of Communication Networks and Information Security (IJCNIS), vol. 9, 2017.

[24] S. M. Chauhan and A. M. Lathigara, "Energy Efficient Multipath OLSR in Mobile Adhoc Networks," International Research Journal of Engineering and Technology (IRJET), vol. 03, 2016. 
[25] V. I. U. Rani and K. T. Reddy, "To Improve the Security of OLSR Routing Protocol Based on Local Detection of Link Spoofing,” International Journal of Science Engineering Technology, IJSEAT, vol. 5, 2017.

[26] S. Prajapati, et al., "Optimizing Performance of OLSR Protocol Using Energy Based MPR Selection in MANET," 2015 Fifth International Conference on Communication Systems and Network Technologies, 2015.

\section{BIOGRAPHIES OF AUTHORS}
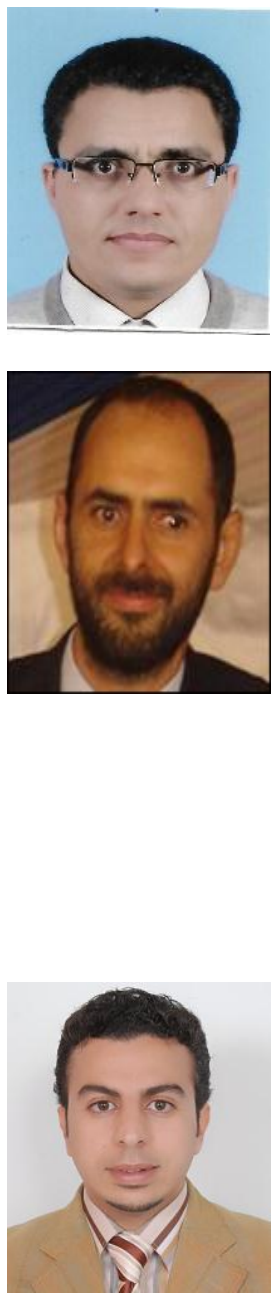

Omar Barki, PhD student in Research Team in Smart Communications - ERSC, E3S Research Center, EMI, Mohammed V University in Rabat, Morocco. He was born in ERRACHIDIA, Morocco in 1975.He received his MST degree in Software Engineering in Sciences and technologies Faculty in 1998, and his Advanced graduate degree in informatics and Telecommunications from Faculty of Sciences of Rabat in 2008, His fields of interest are networks architecture and wireless sensor network.

Zouhair Guennoun, Research Team in Smart Communications - ERSC, E3S Research Center, EMI, Mohammed V University in Rabat, Morocco. Zouhair Guennoun was born in Fès, Morocco in 1964. He received his engineering degree in Electronics and Telecommunications from the Electronics and Electrical Montefiore Institute, ULG Liege, Belgium in 1987; his M.Sc. degree in Communication Systems from the EMI School of Engineering, Rabat, Morocco in 1993; and his PhD degree from the same school in 1996. He visited the Centre for Communication Research (CCR) in Bristol University, UK, during the period of 1990-1994 to prepare a split $\mathrm{PhD}$. His fields of interest are digital signal processing, error control coding, speech and image processing, telecommunication systems, networks architecture and networks security. Prof. Guennoun is an IEEE senior member (member since 1990); and ex-member of the Moroccan IEEE section executive committee. During 1988-1996 he worked as an Assistant Lecturer in the EMI School of engineering, and from 1996 he is working in the same school as a Professor Lecturer. Currently, he is in charge of the research team of Smart Communications - ERSC (formerly known as LEC) as part of the research center in engineering sustainable and smart systems at EMI, University Mohammed $\mathrm{V}$ in Rabat.

Adnane Addaim. He received his master diploma in 2001 and his Ph.D. degree in 2008, both in satellite communication from Mohammadia School of Engineers (EMI), Morocco. From 2002 to 2003, he was employed at SIEMENS.AG, as Transmission Engineer, responsible of the implementation of the supervision network of the Meditelecom GSM Network, in Morocco. From 2004 to 2009, he was teaching assistant at EMI and research engineer at Centre for Space Research and Studies (CRES, EMI) working on research projects dealing with the design of university microsatellite. He is recipient of the first prize of the 2007-2009 best PhD thesis in the field of Sciences and technologies at Mohammed V university. From Mars to August 2010, he was with post-doctoral scholarship at the Satellite Communication and Networking Laboratory (SCNL) at the faculty of engineering, University of Genoa. From 2010 to 2014, he has been Assistant Professor at the ENSA Engineering school kenitra, Morocco. From 2015, he has been an associate professor at the same school. His current research interests include signal processing, communication networks and satellite communication systems. 